\title{
A TRUNCATION PROCESS FOR REDUCTIVE GROUPS
}

\author{
BY JAMES ARTHUR ${ }^{1}$
}

\section{Communicated by J. A. Wolf, January 26, 1977}

Let $G$ be a reductive group defined over $\mathbf{Q}$. Index the parabolic subgroups defined over $\mathbf{Q}$, which are standard with respect to a minimal ${ }^{(0)} P$, by a partially ordered set $\mathcal{I}$. Let 0 and 1 denote the least and greatest elements of $\mathcal{I}$ respectively, so that ${ }^{(1)} P$ is $G$ itself. Given $u \in \mathcal{I}$, we let ${ }^{(u)} N$ be the unipotent radical of ${ }^{(u)_{P}},{ }^{(u)} M$ a fixed Levi component, and ${ }^{(u)} A$ the split component of the center of ${ }^{(u)} M$. Following [1, p. 328], we define a map ${ }^{(u)} H$ from ${ }^{(u)} M(\mathrm{~A})$ to ${ }^{(u)} \mathfrak{a}=\operatorname{Hom}\left(X\left({ }^{(u)} M\right)_{\mathrm{Q}}, \mathbf{R}\right)$ by

$$
e^{\left\langle\chi,(u)_{H(m)}\right\rangle}=|\chi(m)|, \quad \chi \in X\left({ }^{(u)_{M}}\right)_{\mathrm{Q}}, m \in(u)_{M(\mathrm{~A})} .
$$

If $K$ is a maximal compact subgroup of $G(\mathrm{~A})$, defined as in [1, p. 328], we extend the definition of ${ }^{(u)} H$ to $G(\mathrm{~A})$ by setting

$$
{ }^{(u)} H(n m k)={ }^{(u)} H(m), \quad n \in{ }^{(u)} N(\mathbf{A}), m \in{ }^{(u)} M(\mathbf{A}), k \in K .
$$

Identify ${ }^{(0)} \mathfrak{a}$. with its dual space via a fixed positive definite form $\langle$,$\rangle on { }^{(0)} \mathfrak{a}$ which is invariant under the restricted Weyl group $\Omega$. This embeds any $(u)_{\mathfrak{a}}$ into ${ }^{(0)} \mathfrak{a}$ and allows us to regard ${ }^{(u)} \Phi$, the simple roots of $\left({ }^{(u)} P,{ }^{(u)} A\right)$, as vectors in ${ }^{(0)} a$. If $i \leqslant u,{ }^{(v)} P \cap{ }^{(u)} M$ is a parabolic subgroup of ${ }^{(u)} M$, which we denote by $(u) P$ and we use this notation for all the various objects associated with $(u) P$. For example, $\left(\begin{array}{l}(u) \\ a\end{array}\right.$ is the orthogonal complement of ${ }^{(u)} \mathfrak{a}$ in ${ }^{(v)} \mathfrak{a}$ and $\left(\begin{array}{l}v) \\ u\end{array}\right) \Phi$ is the set of elements $\alpha \in{ }^{(v)} \Phi$ which vanish on ${ }^{(u)} a$.

Let $R$ be the regular representation of $G(\mathrm{~A})$ on $L^{2}(Z G(\mathrm{Q}) \backslash G(\mathrm{~A}))$, where we write $Z$ for ${ }^{(1)} A(\mathbf{R})^{0}$, the identity component of ${ }^{(1)} A(\mathbf{R})$. Let $f$ be a fixed $K$ conjugation invariant function in $C_{c}^{\infty}(Z \backslash G(\mathrm{~A}))$. Then $R(f)$ is an integral operator whose kernel is

$$
K(x, y)=\sum_{\gamma \in G(\mathrm{Q})} f\left(x^{-1} \gamma y\right) .
$$

If $u<1$ and $\lambda \in^{(u)} \mathfrak{a} \otimes \mathbf{C}$, let $\rho(\lambda)$ be the representation of $G(\mathbf{A})$ obtained by inducing the representation

$$
(n, a, m) \rightarrow{ }_{(u)} R_{\mathrm{disc}}(m) \cdot e^{\left\langle\lambda,(u)_{H(m)\rangle}\right.}
$$

from ${ }^{(u)} P(\mathrm{~A})$ to $G(\mathrm{~A})$. Here $(u)^{R_{\text {disc }}}$ is the subrepresentation of the representation

AMS (MOS) subject classifications (1970). Primary 22E55; Secondary 32N10.

1 Sloan Fellow. 
of ${ }^{(u)} M(\mathrm{~A})$ on $L^{2}\left({ }^{(u)} A(\mathbf{R})^{0} \cdot(u) M(Q) \backslash^{(u)} M(\mathrm{~A})\right)$ which decomposes discretely. We can arrange that $\rho(\lambda)$ acts on a fixed Hilbert space ${ }^{(u)} \mathrm{H}$ of functions on ${ }^{(u)} N(\mathrm{~A}) \cdot{ }^{(u)} A(\mathrm{R})^{0} \cdot{ }^{(u)} M(\mathrm{Q}) \backslash G(\mathrm{~A})$. If $u=1$, we take ${ }^{(1)} \mathrm{H}$ to be the orthogonal complement of the cusp forms in the subspace of $L^{2}(Z G(\mathbf{Q}) \backslash G(\mathrm{~A}))$ which decomposes discretely.

THEOREM 1. There exist orthonormal bases ${ }^{(u)} \mathcal{B}$ of ${ }^{(u)} H, u \in \mathcal{G}$, such that

$$
K_{E}(x, y)=\sum_{u \in \mathcal{G}} \int_{i(\mathbf{1}) \mathfrak{a}} \sum_{\phi, \phi^{\prime} \in(u) \mathbb{B}}\left(\rho(\lambda, f) \phi^{\prime}, \phi\right) E(\phi, \lambda, x) \overline{E\left(\phi^{\prime}, \lambda, y\right)} d|\lambda|
$$

converges uniformly for $x$ and $y$ in compact subsets of $Z G(Q) \backslash G(\mathrm{~A})$. (Here $E(\phi, \cdot, \cdot)$ is the Eisenstein series associated with $\phi$ as in [3, Appendix II] .) Moreover, $R_{\text {cusp }}(f)$, the restriction of the operator $R(f)$ to the space of cusp forms, is of trace class, and if the Haar measures $d|\lambda|$ on $i_{(1)}^{(u)}$ a are suitably normalized,

$$
\operatorname{tr} R_{\text {cusp }}(f)=\int_{Z G(\mathrm{Q}) \backslash G(\mathrm{~A})}\left(K(x, x)-K_{E}(x, x)\right) d x .
$$

For any $u \in \mathcal{I}$, let ${ }^{(u)} \hat{\Phi}$ be the basis of $\left(_{(1)}^{(1)}\right.$ a which is dual to ${ }^{(u)} \Phi$. We write $|u|$ for the number of elements in ${ }^{(u)} \Phi$ or ${ }^{(u)} \hat{\Phi}$. Let ${ }^{(u)} \hat{\chi}$ be the characteristic function of $\left\{H \in{ }^{(u)} \mathfrak{a}:\langle\mu, H\rangle>0, \mu \in(u) \hat{\Phi}\right\}$. Fix a point $T \in{ }^{(0)} \mathfrak{a}$ such that $\langle\alpha, T\rangle$ is suitably large for each $\alpha \in{ }^{(0)} \Phi$. Motivated by the results of [2, §], we define

$$
\begin{array}{r}
(\Lambda \phi)(x)=\sum_{u \in \mathcal{S}}(-1)^{|u|} \sum_{\delta \epsilon^{(u)}} \sum_{P(\mathrm{Q}) \backslash G(\mathrm{Q})} \int_{(u)_{N(\mathrm{Q}) \backslash}(u)_{N(\mathrm{~A})} \phi(n \delta x) d n} \\
.\left({ }^{(u)} \hat{\chi}\left(^{(u)} H(\delta x)-T\right),\right.
\end{array}
$$

for any continuous function $\phi$ on $Z G(\mathrm{Q}) \backslash G(\mathrm{~A})$. Let $\tilde{k}^{T}(x)$ and $\tilde{k}_{E}^{T}(x)$ be the functions obtained by applying $\Lambda$ to each variable in $K(x, y)$ and $K_{E}(x, y)$ separately, and then setting $x=y$. If $\phi$ is a cusp form, $\Lambda \phi=\phi$. From this it follows that

$$
\tilde{k}^{T}(x)-\tilde{k}_{E}^{T}(x)=K(x, x)-K_{E}(x, x)
$$

THEOREM 2. The functions $\tilde{k}^{T}(x)$ and $\tilde{k}_{E}^{T}(x)$ are both integrable over $Z G(\mathrm{Q}) \backslash G(\mathrm{~A})$, and the integral of $\tilde{k}_{E}^{T}(x)$ equals

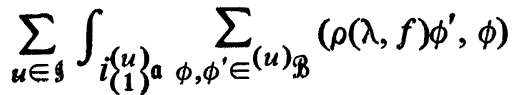

$$
\begin{aligned}
& \int_{Z G(\mathrm{Q}) \backslash G(\mathrm{~A})} \Lambda E(\phi, \lambda, x) \cdot \overline{\Lambda E\left(\phi^{\prime}, \lambda, x\right)} d x d|\lambda| .
\end{aligned}
$$

It should eventually be possible to calculate the integrals in Theorem 2 by extending the methods of $[2, \S 9]$. On the other hand, $\tilde{k}^{T}(x)$ is not a natural truncation of $K(x, x)$. This defect is remedied by the following 
THEOREM 3. The function

$$
\begin{gathered}
k^{T}(x)=\sum_{u \in g}(-1)^{|u|} \sum_{\delta \epsilon^{(u)}} \sum_{P(\mathrm{Q}) \backslash G(\mathrm{Q})} \int_{(u)_{N(\mathrm{~A})}} \sum_{\mu \in(u)} f\left(x^{-1} \delta^{-1} \mu n \delta x\right) d n \\
.{ }^{(u)} \hat{\chi}\left(^{(u)} H(\delta x)-T\right)
\end{gathered}
$$

is integrable over $Z G(\mathbf{G}) \backslash G(\mathbf{A})$. For sufficiently large $T$, the integrals over $Z G(\mathbf{Q}) \backslash G(\mathrm{~A})$ of $k^{T}(x)$ and $\widetilde{k}^{T}(x)$ are equal.

The proofs will appear elsewhere.

\section{REFERENCES}

1. James Arthur, The Selberg trace formula for groups of F.rank one, Ann. of Math. (2) 100 (1974), 326-385. MR 50 \#12920.

2. R. P. Langlands, Eisenstein series, Algebraic Groups and Discontinuous Subgroups, (Proc. Sympos. Pure Math., Boulder, Colo., 1965), Amer. Math. Soc., Providence, R. I., 1966, 235-252. MR 40 \#2784.

3. - On the functional equations satisfied by Eisenstein series, Lecture Notes in Math., vol. 544, Springer-Verlag, Berlin and New York, 1976.

DEPARTMENT OF MATHEMATICS, DUKE UNIVERSITY, DURHAM, NORTH CAROLINA 27706

Current address: School of Mathematics, Institute for Advanced Study, Princeton, New Jersey 08540 